\title{
We need to educate young lung cancer patients about menopause risk
}

\author{
Fahad Faruqi ${ }^{1}$, Elizabeth Cathcart-Rake ${ }^{1}$ \& Kathryn J Ruddy*,1 \\ ${ }^{1}$ Mayo Clinic, Department of Medical Oncology, 200 First Street, SW, Rochester, MN 55901, USA \\ *Author for correspondence: ruddy.kathryn@mayo.edu
}

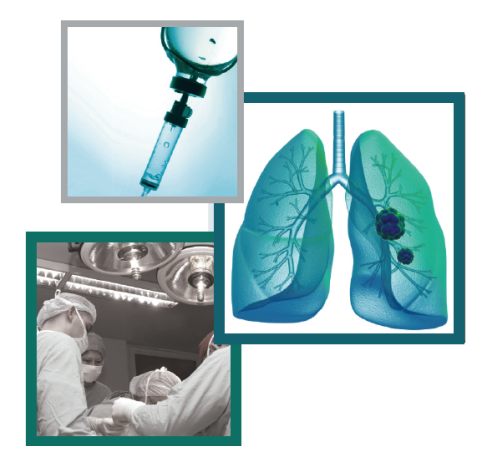

\begin{abstract}
"Those who have not yet completed their desired childbearing at the time of a lung cancer diagnosis should be referred to reproductive endocrinology prior to systemic therapy initiation to consider fertility preservation"
\end{abstract}

First draft submitted: 4 December 2018; Accepted for publication: 17 December 2018; Published online: 12 February 2019

Keywords: fertility • lung cancer $\bullet$ menopause

\section{Background}

Approximately 220,000 Americans are diagnosed with lung cancer each year, leading to 150,000 deaths annually [1]. While the incidence of lung cancer has been decreasing in men, among women the rate has plateaued after increasing for years [1]. A recent study revealed that the incidence of lung cancer in young women has surpassed that of young men between 30 and 54 years of age [2]. These recent trends underscore the importance of understanding the effect of lung cancer treatments on fertility and menopause in young women.

Chemotherapy-associated infertility and premature menopause are known to be frequent concerns among young women diagnosed with other cancers, sometimes impacting cancer-directed therapy decisions and quality of life [3,4]. Amenorrhea (particularly when it is long lasting) is a surrogate for gonadotoxicity in women. Anticancer drugs may diminish fertility and lead to menopause via ovarian atrophy, stromal fibrosis and vascular toxicity [5]. Various types of chemotherapy have been shown to destroy rapidly growing mature ovarian follicles and to induce apoptosis in primordial ovarian follicles [5]. Chemotherapy-induced infertility is most burdensome for younger patients (who have more frequently not completed their desired child-bearing). Female patients treated for cancer during childhood go on to have half as many live births as their sisters who did not get chemotherapy [6]. Alkylating agents such as cyclophosphamide are known to be more gonadotoxic than many other classes of chemotherapeutics and higher doses of cyclophosphamide are most problematic [7]. An analysis of patients with breast cancer enrolled in the International Breast Cancer Study Group Trials V and VI revealed that time to menopause after receiving cyclophosphamide, methotrexate and 5-fluorouracil is dose-dependent; in women younger than 35 who received one or no cycles of cyclophosphamide, methotrexate and 5-fluorouracil, 37\% were menopausal in 5 years, significantly less than the $65 \%$ of women under 35 who received six or seven cycles [8]. Risk of ovarian toxicity increases with age; amenorrhea occurs at least temporarily in more than $80 \%$ of premenopausal women treated with the more modern combination of anthracycline, taxane and cyclophosphamide for early stage breast cancer, but nearly half of women less than 40 eventually resume menses while less than $5 \%$ of those over age 50 do [9]. Similarly, in lymphoma patients, treatment regimens that contain high doses of alkylating agents are associated with the highest risk of menopause and risks are age dependent [10].

While the gonadotoxic effects of many standard treatment regimens for breast cancer and lymphoma are well studied, the risk of menopause and infertility in premenopausal women with lung cancer remains uncertain. In small cell lung cancer, cisplatin plus etoposide is the standard first-line chemotherapy treatment for both limited and extensive stage disease. Platinum-based chemotherapy regimens are frequently first-line choices for non-small-cell lung cancer in both the nonmetastatic and metastatic setting [11]. A patient with metastatic disease often instead receives tyrosine kinase inhibitors (TKIs) as first-line treatment if the tumor has a targetable mutation. Immunotherapy plus chemotherapy is recommended for tumors with low PDL-1 expression, whereas immunotherapy alone is used in patients with high PDL-1 expression [11]. The gonadotoxicity of these drugs is understudied; while cisplatin is 
known to cause significant atresia of ovarian follicles and apoptosis in granulosa cells in rats [12], rates of amenorrhea during and after cisplatin are less clear in humans. It is known that nearly all men who receive cisplatin-based chemotherapy for testicular cancer experience at least temporary azoospermia but $50 \%$ recover by 2 years and $80 \%$ by 5 years [13]. This is similar to the rate and duration of azoospermia during and after cyclophosphamide, doxorubicin, vincristine and prednisone (CHOP) for non-Hodgkin lymphoma [14].

Our recent longitudinal study analyzed the risk of menopause in 182 premenopausal women treated for lung cancer between 1999 and 2016. 85 received platinum-based chemotherapy, while 97 did not. Overall, 55\% of women who received chemotherapy reported becoming menopausal within 2 years of treatment compared with only $31 \%$ of women who received no treatment or targeted therapy [15]. Interestingly, the rate of menopause among women receiving doxorubicin-cyclophosphamide (AC) for breast cancer historically is similar to the rate we identified in these young women who received chemotherapy for lung cancer. While the heterogeneity of populations in the aforementioned studies makes it difficult to draw direct comparisons, these results do suggest that the risk of gonadotoxicity with common platinum-based regimens for lung cancer may mimic that of standard doses of cyclophosphamide given for breast cancer (usually $2400 \mathrm{mg} / \mathrm{m}^{2}$ ).

Our study included too few patients treated with immunotherapy and TKIs alone to assess this. Preclinical studies have revealed that epidermal growth factor receptor expression is a required component of ovarian maturation [16]. Consequently, epidermal growth factor receptor-targeting TKIs could plausibly disrupt normal ovarian function, though larger clinical studies are needed to address these questions.

\section{Treatment of menopausal symptoms}

Diminished ovarian function often leads to the onset of amenorrhea and moderate-to-severe menopausal symptoms after chemotherapy such as hot flashes, sleep disturbance, fatigue and mood changes [17].

For mild vasomotor symptoms, behavior modification such as lowering the room temperature, cooling fans and weight loss have demonstrated efficacy for reducing hot flashes and night sweats [17]. Women who have moderate to severe vasomotor symptoms are often treated with medications. Selective serotonin reuptake inhibitors have been shown in multiple randomized controlled trials to reduce hot flashes by as much as $40 \%$ without a significant increase in adverse events compared with placebo. Gabapentin and pregabalin have also been studied in menopausal women and have demonstrated efficacy in reducing hot flashes. However, they are generally used as second-line drugs in women who do not achieve adequate symptom control with a selective serotonin reuptake inhibitor. Oxybutynin, clonidine and stellate ganglion blockage are other therapies that are potentially promising to reduce severe vasomotor symptoms [17].

In addition to vasomotor symptoms, women who experience premature menopause often have genitourinary symptoms of menopause (GSM) such as vaginal dryness and discomfort with sexual activity. While GSM symptoms significantly impair quality of life, women are unlikely to discuss such symptoms with their healthcare provider [17] and oncologists may be less likely to ask patients with lung cancer about GSM than patients who are receiving endocrine therapies for breast or gynecologic cancers. A number of therapies may improve GSM symptoms, including nonpharmacologic methods, such as vaginal lubricants, topical lidocaine and hyaluronic acid gel or pharmacologic preparations such as vaginal DHEA and estrogen [17]. Because lung cancers do not seem to be hormonally driven (despite evidence of some hormone receptor expression), and because hormone replacement therapy (HRT) does not increase lung cancer incidence, HRT may also be considered (though safety and efficacy of HRT have not been well studied in lung cancer survivors specifically) [18].

\section{Options for fertility preservation}

Because of the risk of early menopause, premenopausal women should be counseled about fertility preservation options prior to initiating lung cancer therapy.

Embryo cryopreservation is the most well-established method of maintaining fertility. However, embryo cryopreservation is only a viable option for women who have a male partner or wish to use a sperm donor. For others, the cryopreservation of mature and immature oocytes may be available [19]. Vitrification, which allows for rapid freezing of oocytes, has led to greater success rates with oocyte cryopreservation than were previously possible [19]. Ovarian tissue cryopreservation remains investigational, though several small studies have reported successful live births with this technique [19].

As the aforementioned options may delay therapy for 2-6 weeks, ovarian function suppression may also be an attractive alternative. There is some controversy about the value of suppressing ovarian function during chemotherapy 
with gonadotropin-releasing hormone agonists to reduce the vulnerability of maturing ovarian follicles to cytotoxic chemotherapy. While these agents appear to increase the rate of ovulation and menses after chemotherapy for breast cancer, no study has shown a definitive increase in the number of live births with the use of ovarian suppression during chemotherapy [19]. Consequently, the American Society of Clinical Oncology (ASCO) guidelines do not include gonadotropin-releasing hormone agonists as recommended fertility preservation technique for patients with cancer [20].

\section{Recommendations}

While we await larger studies of the impact of specific lung cancer therapies on ovarian function, it is important for clinicians to counsel young women that systemic therapy for lung cancer may increase their risk of infertility and premature menopause. Those who have not yet completed their desired childbearing at the time of a lung cancer diagnosis should be referred to reproductive endocrinology prior to systemic therapy initiation to consider fertility preservation (e.g., oocyte and/or embryo cryopreservation). In addition, clinicians should ask about and offer therapies for the genitourinary and vasomotor symptoms of ovarian dysfunction during and after lung cancer treatment.

Financial \& competing interests disclosure

Funded by the TracyStarr Breast Cancer Research Fund. K Ruddy previously owned shares of Pfizer and Merck, which were sold in February 2018.

The authors have no other relevant affiliations or financial involvement with any organization or entity with a financial interest in or financial conflict with the subject matter or materials discussed in the manuscript apart from those disclosed.

No writing assistance was utilized in the production of this manuscript.

\section{Open access}

This work is licensed under the Attribution-NonCommercial-NoDerivatives 4.0 Unported License. To view a copy of this license, visit http://creativecommons.org/licenses/by-nc-nd/4.0/

\section{References}

1. Siegel R, Miller K, Jemal A. Cancer statistics, 2018. CA: Cancer J. Clin. 68(1), 7-30 (2018).

2. Jemal A, Miller K, Ma J. Higher lung cancer incidence in young women than young men in the United States. N. Engl. J. Med. 378(21), 1999-2009 (2018).

3. Ruddy K, Gelber S, Tamimi R. Prospective study of fertility concerns and preservation strategies in young women with breast cancer. J. Clin. Oncol. 32(11), 1151-1156 (2014).

4. Deshpande N, Braun I, Meyer F. Impact of fertility preservation counseling and treatment on psychological outcomes among women with cancer: a systematic review. Cancer 121(22), 3938-3947 (2015).

5. Meirow D, Biederman H, Anderson R, Wallace W. Toxicity of chemotherapy and radiation on female reproduction. Clin. Obstet. Gynecol. 53(4), 727-739 (2010).

6. Schover L. Rates of postcancer parenthood. J. Clin. Oncol. 27(3), 321-322 (2009).

7. Lee M, Gray J, Han H, Plosker S. Fertility and reproductive considerations in premenopausal patients with breast cancer. Cancer Control 17(3), 162-172 (2010).

8. Partridge A, Gelber S, Gelber R, Castiglione-Gertsch M, Goldhirsch A, Winer E. Age of menopause among women who remain premenopausal following treatment for early breast cancer: long-term results from International Breast Cancer Study Group Trials V and VI. Eur. J. Cancer 43(11), 1646-1653 (2007).

9. Swain SM, Land SR, Ritter MW et al. Amenorrhea in premenopausal women on the doxorubicin-and-cyclophosphamide-followed-by-docetaxel arm of NSABP B-30 trial. Breast Cancer Res. Treat. 113(2), 315-320 (2009).

10. Overbeek A, van den Berg M, van Leeuwen F, Kaspers G, Lambalk C, van Dulmen-den Broeder E. Chemotherapy-related late adverse effects on ovarian function in female survivors of childhood and young adult cancer: a systematic review. Cancer Treat. Rev. 53, 10-24 (2017).

11. National Comprehensive Cancer Network. Nonsmall Cell Lung Cancer (2018). www.nccn.org/professionals/physician_gls/pdf/nscl_blocks.pdf

12. Yuksel A, Bildik G, Senbabaoglu F et al. The magnitude of gonadotoxicity of chemotherapy drugs on ovarian follicles and granulosa cells varies depending upon the category of the drugs and the type of granulosa cells. Hum. Reprod. 30(12), 2926-2935 (2015).

13. Colpi G, Contalbi G, Nerva F, Sagone P, Piediferro G. Testicular function following chemo-radiotherapy. Eur. J. Obstet. Gynecol. Reprod. Biol. 113, S2-S6 (2004). 
14. Pryzant RM, Meistrich ML, Wilson G, Brown B, McLaughlin P. Long-term reduction in sperm count after chemotherapy with and without radiation therapy for non-Hodgkin's lymphomas. J. Clin. Oncol. 11(2), 239-247 (1993).

15. Cathcart-Rake E, Ruddy K, Gupta R et al. Amenorrhea after lung cancer treatment. Menopause doi: 10.1097/GME.000000000001199 (2018) (Epub ahead of print).

16. Jamnongjit M, Gill A, Hammes SR. Epidermal growth factor receptor signaling is required for normal ovarian steroidogenesis and oocyte maturation. Proc. Natl Acad. Sci. USA 102(45), 16257-16262 (2005).

17. Faubion S, Loprinzi C, Ruddy K. Management of hormone deprivation symptoms after cancer. Mayo Clin. Proc. 91(8), 1133-1146 (2016).

18. Bae J, Kim E. Hormonal replacement therapy and the risk of lung cancer in women: an adaptive meta-analysis of cohort studies. J. Prev. Med. Public Health 48(6), 280-286 (2015).

19. Donnez J, Dolmans M. Fertility preservation in women. N. Engl. J. Med. 377(17), 1657-1665 (2017).

20. Oktay K, Harvey B, Loren A. Fertility preservation in patients with cancer: ASCO clinical practice guideline update summary. J. Oncol. Pract. 14(6), 381-385 (2018). 